\title{
A new chemical concept: Shape chemical potentials
}

\author{
Garnet Kin-Lic Chan and Nicholas C. Handy \\ Department of Chemistry, University of Cambridge, Lensfield Road, CB2 1EW, United Kingdom
}

(Received 16 April 1998; accepted 19 June 1998)

\begin{abstract}
Within the density functional formalism, we introduce the shape chemical potential $\mu_{i}^{n}$ for subsystems, which in the limiting case of point subsystems, is a local chemical potential $\mu^{n}(\mathbf{r})$. It describes the electron withdrawing/donating ability of specified density fragments. The shape chemical potential does not equalize between subsystems, and provides a powerful new method to identify and describe local features of molecular systems. We explore the formal properties of $\mu_{i}^{n}$ especially with respect to discontinuities, and reconcile our results with Sanderson's principle. We also perform preliminary calculations on model systems of atoms in molecules, and atomic shell structure, demonstrating how $\mu_{i}^{n}$ and $\mu^{n}(\mathbf{r})$, identify and characterize chemical features as regions of different shape chemical potential. We present arguments that shell structure, and other chemical features, are not ever obtainable within Thomas-Fermi-type theories. (C) 1998 American Institute of Physics. [S0021-9606(98)30536-X]
\end{abstract}

\section{INTRODUCTION}

The development of density functional theory as a description of the electronic structure of atoms and molecules continues at a rapid pace. The density functional formulation is conceptually appealing, as it resembles classical thermodynamic theory. ${ }^{1}$ Central to an understanding of open systems in thermodynamics is the system chemical potential $\mu_{0}$. In density functional theory, this arises naturally as a Lagrange multiplier for the particle number, in the Euler equation

$$
\frac{\delta}{\delta \rho}[E[\rho]-\mu N]=0,
$$

where all symbols have their usual meaning, and throughout this work, the external potential $v(\mathbf{r})$, is held fixed, and derivatives (unless otherwise stated) are evaluated at the ground state density. The system chemical potential corresponds to a choice of Lagrange multiplier $\mu_{0}=\partial E_{0} / \partial N$, constant through space, where $E_{0}$ is the ground-state energy. (We say "a choice of Lagrange multiplier" because for energy functionals that are nonsmooth, as considered in this work, $\mu$ is not uniquely defined.) Following Parr, Donnelly, Levy, and Palke, ${ }^{2}$ the link with structural chemistry is made, by also identifying $\mu_{0}$ as $-\chi$, where $\chi$ is the system electronegativity.

To describe chemical behavior in density functional theory, there are a large number of natural quantities as well as $\mu_{0}$. We have further derivatives of the energy, the hardness $\eta$ and its inverse $\sigma$, the softness, Fukui functions $f(\mathbf{r})$, and many others. The resemblance of the density functional formalism to classical thermodynamics ensures that these are microscopic analogs of thermodynamic quantities. They describe changes of ground-state, arise naturally as derivatives of ground-state quantities, such as $E_{0}$, and are often global.

However, such quantities do not give a complete description of chemistry. Throughout chemistry, subsystems are of great importance. Subsystems exist on all scales: rang- ing from functional groups to orbitals. As they often exist as local features, they are not properly described using global quantities like $\mu_{0}$. A related point, is that the chemistry of subsystems is not always concerned with changes in the ground-state. Instead, chemical reactions involving specific localized subsystems (such as functional groups) generally refer to changes to metastable states. Chemically useful quantities must be able to describe such behavior.

It is imperative to develop local versions of global thermodynamic quantities. In recent years, significant progress has been made. ${ }^{3,4}$ However, a local chemical potential has yet to be explored theoretically. We often talk of subsystems in molecules as possessing a certain electronegativity, thus there is a strong utilitarian basis for some form of local chemical potential. This quantity is the subject of our study in this work.

Donnelly and Parr, ${ }^{5}$ and later Janak, ${ }^{6}$ introduced a chemical potential quantity for orbitals, as the partial derivative of the energy with respect to the occupation numbers of the orbitals, $\partial E / \partial n_{i}$ (holding all other occupation numbers, and the shapes of all orbitals, fixed).

We build on this idea. In Sec. II, we define a general subsystem shape chemical potential $\mu_{i}^{n}$ for any density fragment. It is somewhat different to the usual chemical potential, as this quantity refers to the rate of change of energy with occupation number, holding the shape of the subsystem density (and also the density outside the subsystem) fixed. In the limit of a point subsystem, we identify the shape chemical potential as the local chemical potential $\mu^{n}(\mathbf{r})$.

In Sec. III, we lay the groundwork for the use of $\mu_{i}^{n}$, by first considering its formal properties. For well-behaved, continuously differentiable (smooth) energy functionals, the subsystem chemical potential should be identical to the system chemical potential $\mu_{0}$, at the equilibrium density. However, this is not the case, because we work throughout at zero temperature, where the exact energy functional is not smooth. In the first part, we discuss the resulting situation. Through the density functional theory of open systems, ${ }^{7,8}$ we 
explore the discontinuities in $\mu_{i}^{n}$, and obtain understanding of the global $\mu_{0}$.

In the second part we consider Sanderson's principle ${ }^{9}$ which states that the chemical potential of density fragments should equalize, on the formation of a molecule. This is at odds with a subsystem chemical potential that varies between subsystems, or equally a local chemical potential which is not constant through space. We reconcile our subsystem chemical potential with Sanderson's principle, and resolve a paradox first raised by Donnelly and Parr, ${ }^{1,5}$ namely why $\mu_{i}^{n}$, does not equalize on an orbital by orbital basis in Kohn-Sham theory.

We next propose the use of the subsystem chemical potential to understand important aspects of chemistry, in Sec. IV. While the chemical picture obtained will clearly depend on the partitioning into subsystems, we suggest that we can use the local chemical potential itself, to identify useful partitionings into chemically interesting subunits of molecules. Changing the "grain size" of the subsystem shape chemical potential allows one to change the scale and resolution with which one describes the molecule; with a small grain size we may be able to identify shell structure, while going to larger grain sizes, we may see the individual atoms only. To verify these ideas we calculate shape chemical potentials for several model systems. The picture that emerges, while only a preliminary one, is suggestive and intuitive, and new insights into the nature of atoms in molecules, and the origin of atomic shell structure, are revealed.

If the results of this work for model systems extend to real systems, there are important implications for ThomasFermi theories. We suggest why Thomas-Fermi-type theories will never give shell structure, or other interesting chemistry. The importance of derivative discontinuities in $E[\rho, v]$ for chemistry is stressed.

We summarize our findings and propose some future applications in Sec. V.

\section{THE SHAPE CHEMICAL POTENTIAL}

Consider $m$ subsystems $\rho_{i}(\mathbf{r})$ (which may be overlapping) of the density $\rho(\mathbf{r})$, such that

$$
\sum_{i}^{m} \rho_{i}(\mathbf{r})=\rho(\mathbf{r})
$$

Further define quantities

$$
\begin{aligned}
& n_{i}=\int \rho_{i}(\mathbf{r}) d \mathbf{r}, \\
& \rho_{i}(\mathbf{r})=n_{i} \Phi_{i}(\mathbf{r}),
\end{aligned}
$$

where $n_{i}$ are the occupation numbers, and $\Phi_{i}(\mathbf{r})$ are the shape functions of the subsystems. The shape function is normalised such that $\int \Phi_{i}(\mathbf{r}) d \mathbf{r}=1 . \Phi_{i}(\mathbf{r})$ contains information on the shape of the density of the subsystem. For example, in the Hartree-Fock resolution of the density into orbitals $\psi_{i}(\mathbf{r})$, then $\Phi_{i}(\mathbf{r})=\psi_{i}^{2}(\mathbf{r})$. The exact $E[\rho, v]$, given by $\min _{\Psi \rightarrow \rho}\langle\Psi|\hat{H}| \Psi\rangle_{v(\mathbf{r})}$, as a functional of $\rho(\mathbf{r})$ and $v(\mathbf{r})$, is also a functional of the quantities $n_{i}, \Phi_{i}(\mathbf{r}), v(\mathbf{r})$, [through relations (2) and (4)].
Next, define the shape chemical potential of the subsystem $i, \mu_{i}^{n}$,

$$
\mu_{i}^{n}=\frac{\partial E}{\partial n_{i_{\mathbf{n}^{\prime} \Phi}}},
$$

where the subscript $\mathbf{n}^{\prime} \boldsymbol{\Phi}$, indicates that the occupation numbers of all subsystems other than $i$, and the shape functions of all subsystems, are held fixed. This is the response of the energy to number changes in a subsystem, maintaining the shape of the subsystem (and the density outside the subsystem) fixed. $\mu_{i}^{n}$ may be regarded as the conjugate variable to $n_{i}$, as reflected in the notation. (This remark is due to Hansen. ${ }^{10}$ )

We make contact with the orbital chemical potentials of Janak's theorem, ${ }^{6}$ and the analysis of Donnelly and Parr, ${ }^{5}$ as they are just examples of the above. Partition the density $\rho(\mathbf{r})$ into a set of $m$ orthonormal orbitals $\psi_{i}(\mathbf{r})$, through

$$
\rho(\mathbf{r})=\sum_{i}^{m} n_{i} \psi_{i}^{2}(\mathbf{r}) \text {. }
$$

To obtain Janak's theorem, choose $m \geqslant N, 0 \leqslant n_{i} \leqslant 1$, and the orbitals such that for a given set $\left\{n_{i}\right\}$, they minimize the generalized kinetic energy $T_{J}$ given by

$$
T_{J}[\rho]=-\frac{1}{2} \sum_{i}^{m} n_{i}\left\langle\psi_{i}\left|\nabla^{2}\right| \psi_{i}\right\rangle,
$$

subject, of course, to yielding the density through (6). The results of Donnelly and Parr, are more general as they refer to density matrices, but may be obtained in the same fashion. They consider the case where $m \gg N$, and the orbitals minimize $E[\gamma]$, the first order density matrix $\gamma$ functional, where $\gamma=\sum_{i}^{m} n_{i} \psi(\mathbf{r}) \psi^{*}\left(\mathbf{r}^{\prime}\right)$. Then we can define orbital chemical potentials in both the Janak, and Donnelly and Parr picture through $^{5,6}$

$$
\begin{aligned}
\frac{\partial E}{\partial n_{i_{\mathbf{n}^{\prime} \boldsymbol{\Phi}}}} & =\epsilon_{i} \\
& =\mu_{i}^{n} .
\end{aligned}
$$

In the Janak case $\epsilon_{i}$ is the eigenvalue of $\psi_{i}$ in the orbital equation

$$
\left[-\frac{1}{2} \nabla^{2}+v_{\text {eff }}(\mathbf{r})\right] \psi_{i}(\mathbf{r})=\epsilon_{i} \psi_{i}(\mathbf{r}),
$$

where $v_{\text {eff }}(\mathbf{r})=\delta\left(E[\rho]-T_{J}[\rho]\right) / \delta \rho$ (see $\mathrm{Janak}^{6}$ for details). In the density matrix case of Donnelly and Parr, $\epsilon_{i}$ occurs in the coupled integral equation

$$
\int d \mathbf{r} F\left[\gamma, \mathbf{r}^{\prime}, \mathbf{r}\right] \psi_{i}(\mathbf{r})=\epsilon_{i} \psi_{i}\left(\mathbf{r}^{\prime}\right),
$$

where the kernel $F\left[\gamma, \mathbf{r}^{\prime}, \mathbf{r}\right]=\delta E[\gamma] / \delta \gamma$ (see Donnelly and Parr for a full derivation). ${ }^{5}$ In the special case when $\psi_{i}$ are the natural orbitals, $\mu_{i}^{n}$ is the same for all orbitals, and is just $\mu_{0}$, the system chemical potential. ${ }^{5}$

There is a loosely analogous quantity to $\mu_{i}^{n}$ in macroscopic thermodynamics, suggested by Donnelly and Parr ${ }^{5}$ in relation to the orbital chemical potentials. If we take the case of subsystems as different phases of a single component, the 
electron fluid, then (5) is analogous to the definition of the chemical potential of a particular phase $\Phi_{i}$. However, the analogy cannot be pursued too far. $E[\rho, v]$ is not necessarily a ground state energy, and has infinite degrees of freedom, unlike the macroscopic $E(N, V)$.

We shall be particularly interested in a limiting case of $\mu_{i}^{n}$. In the limit when the subsystems shrink to points of infinitesimal volume, then $M \rightarrow \infty, \Phi_{i}(\mathbf{r}) \rightarrow \delta\left(\mathbf{r}^{\prime}-\mathbf{r}\right)$, and $n_{i} \rightarrow \rho(\mathbf{r})$. The energy is no longer dependent on $\Phi_{i}(\mathbf{r})$, and $i$ is a continuous variable $\mathbf{r}$. We introduce the local chemical potential $\mu^{n}(\mathbf{r})$,

$$
\mu^{n}(\mathbf{r})=\frac{\partial E}{\partial \rho(\mathbf{r})_{\boldsymbol{\rho}\left(\mathbf{r}^{\prime}\right)}},
$$

where we hold the density at all places other than $\mathbf{r}$, fixed.

We note the following properties of $\mu_{i}^{n}$ and $\mu^{n}(\mathbf{r})$ in a naive fashion. From the definition, if the subsystems are chosen to be localized, then the subsystem shape chemical potentials are dependent on, and thereby a characteristic of the choice of localised regions (although a priori we cannot yet conclude if $\mu_{i}^{n}$ is in fact identical in all subsystems). Furthermore, the shape chemical potential of a subsystem refers to the rate of change in energy on uniformly scaling the density of the subsystem, which is not in general just a change along the path of ground state densities. The shape chemical potential shows promise as a chemical concept. Formal properties of $\mu_{i}^{n}$ and $\mu^{n}(\mathbf{r})$ are discussed in detail next.

\section{FORMAL PROPERTIES}

\section{A. System vs shape chemical potentials}

We can relate $\mu_{i}^{n}(\mathbf{r})$ to $\delta E / \delta \rho(\mathbf{r})$ by manipulating functional derivatives. Consider some small density perturbation, resolved thus

$$
\delta \rho(\mathbf{r})=\sum_{i}\left(d n_{i} \Phi_{i}(\mathbf{r})+\delta \Phi_{i}(\mathbf{r}) n_{i}\right)
$$

Then from the definition of the functional derivative

$$
\begin{aligned}
d E & =\int\left[\frac{\delta E}{\delta \rho(\mathbf{r})}\right] \delta \rho(\mathbf{r}) d \mathbf{r} \\
& =\int\left[\frac{\delta E}{\delta \rho(\mathbf{r})}\right]\left[\left(\sum_{i} d n_{i} \Phi_{i}(\mathbf{r})+\delta \Phi_{i}(\mathbf{r}) n_{i}\right)\right] d \mathbf{r},
\end{aligned}
$$

we have

$$
\mu_{i}^{n}=\int\left[\frac{\delta E}{\delta \rho(\mathbf{r})}\right] \Phi_{i}(\mathbf{r}) d \mathbf{r} .
$$

At equilibrium, $\delta E / \delta \rho(\mathbf{r})=\mu$, and so from Eq. (16), the shape chemical potential takes on the significance of a Lagrange multiplier in the Euler equation.

Do we expect $\mu^{n}(\mathbf{r})$ and $\mu_{i}^{n}$ to be equivalent to $\mu_{0}$ ? For a continuously differentiable functional $E[\rho, v]$ (defined for all $N$ ), that satisfies Eq. (1), $\mu=\mu_{0}$, and from Eq. (16), it follows that $\mu_{i}=\mu_{0}=\mu_{i}^{n}$. However, at zero temperature and integer particle number, to which we restrict ourselves in this work, the answer is in general, no.
It is known that the zero temperature extension of $E[\rho, v]$ to all real particle numbers, by Perdew, Parr, Levy, and Balduz (which is the only "reasonable", extension ${ }^{11}$ ), is not continuously differentiable with respect to $\rho(\mathbf{r})$ at integer $N$. In particular, the change in energy from certain number conserving variations $(d N=0)$, for example, those that transfer $d N$ electrons from atom $\mathrm{X}$ to a well separated atom $\mathrm{Y}$, in the system $\mathrm{XY}$, is of order $\left.|d N|\right|^{8,12}$ Although we write $\delta E / \delta \rho(\mathbf{r})=\mu$, there is more than one Lagrange multiplier that satisfies the Euler equation, and in general $\mu \neq \mu_{0}$. This we illustrate now.

Recall that $\mu_{0}=\partial E_{0} / \partial N$, where we now take the derivatives at integer particle number. This is the rate of change in the energy taking an infinitesimal step $\epsilon$ along the path of ground state $N \rightarrow N+1$ densities, $\left[\rho_{N}(\mathbf{r}) \rightarrow(1-\boldsymbol{\epsilon}) \rho_{N}(\mathbf{r})\right.$ $+\epsilon \rho_{N+1}(\mathbf{r})$, where $\rho_{N}$ is the ground state density of the $N$-electron system $\left.{ }^{8}\right]$, thus

$$
\begin{aligned}
\mu_{0} & =\int\left[\frac{\delta E}{\delta \rho(\mathbf{r})}\right]\left[\frac{\delta \rho(\mathbf{r})}{\delta N}\right] d \mathbf{r} \\
& =\int\left[\frac{\delta E}{\delta \rho(\mathbf{r})}\right]\left[\rho_{N+1}(\mathbf{r})-\rho_{N}(\mathbf{r})\right] d \mathbf{r},
\end{aligned}
$$

and since Eq. (1) is satisfied, $\mu=\mu_{0}$. However in the general case, we may choose some other path of density change. For example, for $\mu_{i}^{n}=\partial E / \partial n_{i_{\mathbf{n}^{\prime} \boldsymbol{\Phi}}}$, we are considering an infinitesimal step along the path $\rho_{N}(\mathbf{r}) \rightarrow \rho_{N}(\mathbf{r})+\epsilon \Phi_{i}(\mathbf{r})$. Subtracting the changes along the two paths, if the number conserving variation $\epsilon\left(\rho_{N+1}(\mathbf{r})-\rho_{N}-\Phi_{i}(\mathbf{r})\right)$ causes a first order variation in the energy, then $\mu_{i}^{n}-\mu_{0} \neq 0$. In essence, the functional derivative $\delta E / \delta \rho(\mathbf{r})$, at the ground-state density, depends on the path $\delta \rho(\mathbf{r})$ along which we approach the ground-state density.

In fact, since the exact functional satisfies the variational condition $^{1}$

$$
E\left[\rho \pm d n_{i} \Phi_{i}, v\right] \geqslant E_{0}\left[N \pm d n_{i}, v\right]
$$

[where Eq. (19) follows when we recall $\int d n_{i} \Phi_{i}(\mathbf{r}) d \mathbf{r}=d n_{i}$, and denoting left derivative and right derivative energy quantities by - and + , respectively, we conclude

$$
\begin{aligned}
& \mu_{i_{-}}^{n} \leqslant \mu_{0_{-}}, \\
& \mu_{i_{+}}^{n} \geqslant \mu_{0_{+}} .
\end{aligned}
$$

When the subsystem encompasses the entire system, we give the shape chemical potential the special term isomorphic chemical potential, denoted $\mu^{N}$. This is not generally identical to $\mu_{0}$. This is proved by a reductio ad absurdum, counterexample.

Consider the case of well separated atoms $\mathrm{X}$ and $\mathrm{Y}$, with first electron affinities $A_{\mathrm{X}}$ and $A_{\mathrm{Y}}$, respectively, and assume also, without loss of generality, that $A_{\mathrm{Y}}<A_{\mathrm{X}}$. Our proposition, to be proved false, is

$$
\mu_{0}=\mu^{N} \text {. }
$$

The first order energy change in $E[\rho, v]$, associated with a change $+d N$ along the path of ground-state densities, adding density to $\mathrm{Y}$ ( since $A_{\mathrm{Y}}<A_{\mathrm{X}}$ ), is

$$
d E=\mu_{0} d N=A_{Y} d N=\mu_{N} d N .
$$


We use the value of $\mu_{0}$ predicted by the density functional theory of open systems, ${ }^{1,8}$ and the last equality follows from Eq. (22). However, $\mu^{N}$ is also associated with the first order energy change, of uniformly scaling the density of the system, holding its shape fixed. This corresponds to a change of particles on atom $\mathrm{X}, d N_{\mathrm{X}}=N_{\mathrm{X}} d N /\left(N_{\mathrm{X}}+N_{\mathrm{Y}}\right)$, and similarly for atom Y. From our proposition, the isomorphic chemical potentials of $\mathrm{X}$ and $\mathrm{Y}$ separately, are also their system chemical potentials, $A_{\mathrm{X}}$ and $A_{\mathrm{Y}}$. The total energy change associated with scaling the density, is then

$$
d E=\frac{N_{\mathrm{X}} A_{\mathrm{X}}+N_{\mathrm{Y}} A_{\mathrm{Y}}}{N_{\mathrm{X}}+N_{\mathrm{Y}}}
$$

giving $\mu^{\mathrm{N}}=\left(N_{\mathrm{X}} A_{\mathrm{X}}+N_{\mathrm{Y}} A_{\mathrm{Y}}\right) /\left(N_{\mathrm{X}}+N_{\mathrm{Y}}\right)$, and thus our proposition, that $\mu^{N}=\mu_{0}$ is false, completing the proof.

The precise difference between the isomorphic chemical potential $\mu^{N}$ and $\mu_{0}$, for isolated atoms and molecules, is of some importance if the shape chemical potential is to retain any link with the system chemical potential, and thereby with experimental quantities such as the ionization energy and electron affinity. This can only be answered by explicit computation.

It remains only to consider the relative values of $\mu_{i}^{n}$ between the subsystems. Is $\mu_{i}^{n}=\mu_{j}^{n}$ ? This we answer in the next section.

\section{B. Chemical potential equalization}

In macroscopic thermodynamics, equalization of chemical potentials represents a driving force to equilibrium. The microscopic embodiment of this law is stated in Sanderson's principle, ${ }^{9}$

"When two or more atoms initially different in chemical potential (electronegativity) combine chemically their (electronegativities) become equalized in the molecule."

This statement also led to the identification of the chemical potential with the Mulliken electronegativity. ${ }^{2,5}$

Does $\mu_{i}^{n}$ obey Sanderson's principle? If the subsystem chemical potential $\mu_{i}^{n}$ is considered as a continuous function of $n_{i}$, and if $\mu_{i}^{n}$ does not equalize between the subsystems, there will exist first order density changes which lower the energy, by transfer of particles from a region $i$ to a region $j$, where $\mu_{i}^{n}>\mu_{j}^{n}$.

However, it follows from Eqs. (20) and (21) of the last section that $\mu_{i}^{n}$ cannot be a smooth continuous function of $n_{i}$, if $\mu_{0}$ is discontinuous with respect to $N$, and if $E\left[n_{i}, \Phi_{i}, v\right]$ satisfies a variational theorem. Instead, subtracting Eq. (21) from Eq. (20) (for general subsystems $i$ and $j$ ) the discontinuity in $\mu_{i}^{n}$ obeys

$$
\mu_{j_{+}}^{n}-\mu_{i_{-}}^{n} \geqslant \mu_{0_{+}}-\mu_{0_{-}},
$$

with $i$ and $j$ being any index. Further, from the (experimental) convexity of $E_{0}[N, v]$ in $N,{ }^{8}$ the right-hand side is positive semidefinite, $I-A$ (the difference between the system ionization energy and electron affinity) at the integer, and zero off the integer. Using the conditions on the left- and right-hand derivatives separately, Eqs. (20) and (21), Eq. (25) is an equality only when $\mu_{i_{+}}^{n}=\mu_{0_{+}}$, and $\mu_{i_{-}}^{n}=\mu_{0_{-}}$. As shown in the previous section, even separately, the latter two conditions are not generally satisfied, and even off the integer, it is only in very special cases that the discontinuity vanishes.

Thus $\mu_{i}^{n}$ does not in general obey Sanderson's principle, and is a characteristic quantity of each subsystem. At integer particle numbers, any transfer of particles $d n$ between subsystem $i$ and $j$ leads to a first order change in the energy $d E \geqslant(I-A)|d n|$. This is why Kohn-Sham eigenvalues, proven to be $\partial E / \partial n_{i}$, do not equalize at the equilibrium density, resolving the question posed by Donnelly and Parr. $^{5,13}$

The very fact that $\mu_{i}^{n}$ does not equalize between subsystems, even at the ground-state density, is precisely what gives $\mu_{i}^{n}$ value as a chemical descriptor. The chemical potential as a driving force to equilibrium is a secure and valuable picture, but there are advantages also in the shape chemical potential $\mu_{i}^{n}$, as a quantity which characterizes the electron donating/accepting ability of a particular density fragment, allowing us to describe the chemistry of a subsystem as opposed to that of the system as a whole.

For example, consider again the case of two atoms $\mathrm{X}$ and $\mathrm{Y}$, well separated, with $A_{\mathrm{Y}}<A_{\mathrm{X}}$. The system chemical potentials of $\mathrm{X}$ and $\mathrm{Y}, \partial E_{0} / \partial N_{\mathrm{X}}$ and $\partial E_{0} / \partial N_{\mathrm{Y}}$ (taking right derivatives, say), equalize at $A_{\mathrm{Y}}$, as an added electron will always go to the atom with the lowest electron affinity, even at infinite separation. ${ }^{1,8}$ This tells us no information about the atomic constituents of the system. Taking $\mathrm{X}$ and $\mathrm{Y}$ as separate subsystems, however, the subsystem shape chemical potentials $\mu_{\mathrm{X}}^{n}$ and $\mu_{\mathrm{Y}}^{n}$ will not equalize, and tend instead to the respective isomorphic chemical potentials of the isolated atoms, as $\mathrm{X}$ and $\mathrm{Y}$ are separated.

This illustrates the main theme of the following work. The shape chemical potential is different in different subsystems of molecules. Does this correlate in a useful way with chemistry? We proceed to consider this question in the next section.

\section{USING THE SHAPE CHEMICAL POTENTIAL TO DESCRIBE CHEMISTRY}

We wish to use the shape chemical potential to describe chemistry. Clearly, the picture that will emerge, will depend on our partitioning of the system into subsystems. If we choose a Kohn-Sham orbital resolution of the density, then the shape chemical potentials are just the orbital eigenvalues, and we recover a molecular orbital theory picture of the molecule. There are many other choices of subsystems.

Indeed, the identification and characterization of useful molecular subunits is what practicing chemistry is all about. We suggest that we can use the subsystem shape chemical potential itself, to do this. We propose the following theoretical experiment-consider only one particular type of subsystem; a grain of finite volume, which may be shrunk in a continuous fashion. In linear systems, the grain represents some connected interval of space; in the spherical systems, we take the grain to be a uniform spherical shell of finite thickness. In the limit of a vanishing grain, the grain shape chemical potential is the local chemical potential. 

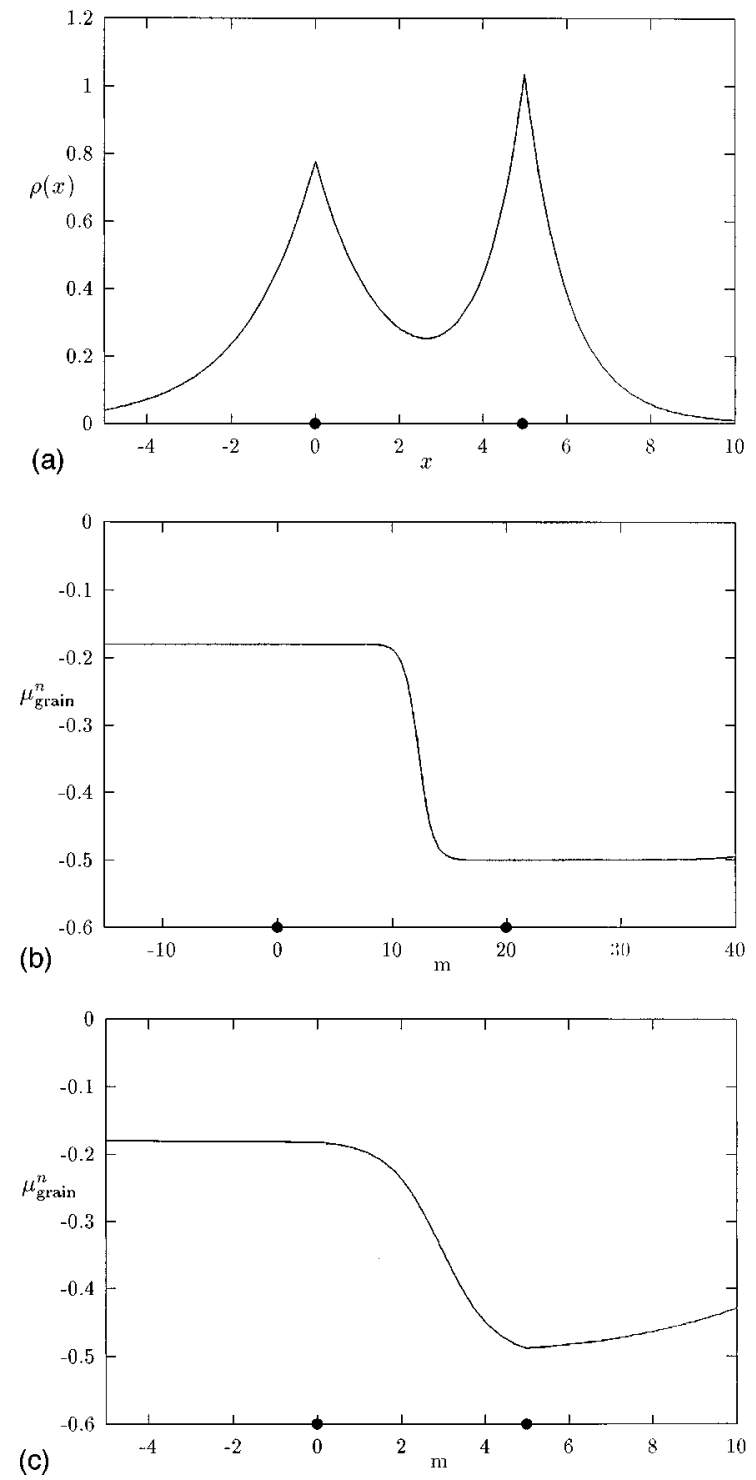
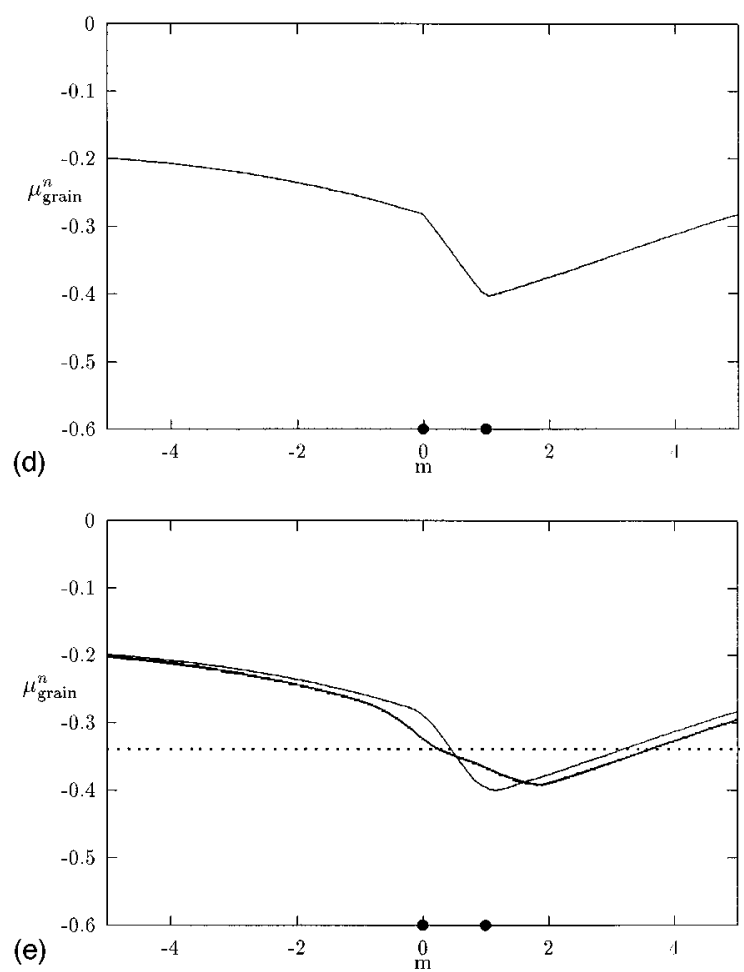

FIG. 1. (a) One dimensional diatomic density $\rho(x)$, with bond length $l=5$. (b) One dimensional diatomic $\mu_{\text {grain }}^{n}$, bond length $l=20$, grain size $g=0.001$. (c) One dimensional diatomic $\mu_{\text {grain }}^{n}$, bond length $l=5$, grain size $g=0.001$. (d) One dimensional diatomic $\mu_{\text {grain }}^{n}$, bond length $l=1$, grain size $g=0.001$. See Sec. IV A. (e) One dimensional diatomic $\mu_{\text {grain }}^{n}$, bond length $l=1$, with varying grain size (thin line, $g=0.25$; thick line, $g=1$; dotted line, $g=50$ ). In the above, the positions of the atoms are given by the circles. See Sec. IV A.

By moving the grain along in space, that is along the line of symmetry for linear systems, and along the radius for spherical systems, and evaluating the grain shape chemical potential at each point in space, we look to see if we can identify the various chemical features of the system. For vanishingly small grain sizes, which correspond to the evaluation of the local chemical potential, we hope to pick up a detailed picture of systems, for example, the shell structure. With a larger grain, say of roughly atomic size, we hope to see the system broken down into in terms of larger subunits, such as atoms.

As our first investigation, we conduct this experiment in a set of model systems, for which all calculations may be performed analytically. These model systems are noninteracting systems, and constitute a one dimensional two electron diatomic, a two electron atom, and a model piecewise exponential density multielectron atom.

\section{A. One dimensional, two electron diatomic}

We choose this model of a diatomic after Perdew, ${ }^{14}$ and our investigations here are related to his investigations of the effective potential in these systems. It consists of two one dimensional atoms centered at $x=0$ and $x=l$, which form the natural subsystems of the molecule. By studying this model, we hope to capture the flavor of the traditional "atoms-in-molecules" approach, using the shape chemical potential.

The density is constructed as

$$
\rho(x)=c\left(\sqrt{a} e^{-a|x|}+e^{-|x-l|}\right)^{2},
$$

where $c$ normalizes, $\rho(x)$ to 2 electrons. $a$ may be regarded as an "electronegativity," while $l$ is just the bond length. This density is plotted in Fig. 1(a), for $a=0.6$ and $l=5$, where we work within atomic units. 
The energy of a noninteracting 1D density where $0 \leqslant N$ $\leqslant 2$ is given by

$$
E[\rho, v]=-\frac{1}{2} \int \rho(x)^{1 / 2} \frac{d^{2}}{d x^{2}} \rho(x)^{1 / 2} d x+\int \rho(x) v(x) d x,
$$

where the external potential $v(x)$ is chosen to cancel the contributions of the cusp derivative discontinuity to the kinetic energy,

$$
\begin{aligned}
v(x)= & -\frac{1}{4 \rho(0)}\left[\left(\frac{d \rho}{d x}\right)_{x=0,+}-\left(\frac{d \rho}{d x}\right)_{x=0,-}\right] \delta(x) \\
& -\frac{1}{4 \rho(l)}\left[\left(\frac{d \rho}{d x}\right)_{x=l,+}-\left(\frac{d \rho}{d x}\right)_{x=l,-}\right] \delta(x-l) .
\end{aligned}
$$

We take the subsystem as a grain centred at $x=m$, with boundaries at $m+g, m-g$ on either side, where $g$ is the grain size parameter. The occupation number of the grain (as a function of its position $m$ ) is thus

$$
n(m)=\int_{m-g}^{m+g} \rho(x) d x .
$$

The chemical potential of the grain as a function of its position $m, \mu_{\text {grain }}^{n}(m)$ (taking left derivatives), after a little algebra, is

$$
\begin{aligned}
\mu_{\text {grain }}^{n}(m)= & \frac{\partial E}{\partial n_{m}} \\
= & \frac{1}{n(m)} \int_{m-g}^{m+g}-\frac{1}{2} \rho(x)^{1 / 2} \frac{d^{2}}{d x^{2}} \rho(x)^{1 / 2} \\
& +\rho(x) v(x) d x .
\end{aligned}
$$

We see that it is an energy density quantity. ${ }^{15}$

We start the investigation with a small grain size, $g$ $=0.001$ (with all quantities in atomic units), which corresponds (approximately) to calculating the local chemical potential. Plotted in Figs. 1(b)-1(d) is $\mu_{\text {grain }}^{n}(m)(g=0.001)$ against $m$, with the bond distance $l$ as 20,5 , and 1 units, for a heteronuclear diatomic with $a=-0.6$.

When the atoms are widely separated, their atomic nature is clearly manifested as two regions of distinct, constant local chemical potential, centered around each atom, with the size of the region of constant potential increasing as the atoms are drawn apart. There is a sharp change in the local chemical potential in the region of maximum overlap between the two "atomic" densities, which can be identified as the "bonding"' region. In Figs. 1(c) and 1(d), as the atoms are drawn together, they begin to lose their separate identity. However, three distinct regions are still apparent; the local chemical potentials assume gentle gradients in each of the two atomic regions, and size of the "bonding region" increases. At bond length $l=5$, the bonding region coincides well with the region of maximum overlap between atomic densities in Fig. 1(a). An additional feature, which may or may not be an artifact of this model, is that at infinity, the local chemical potential tends to the HOMO eigenvalue (evaluated here as $\frac{1}{2}\left\langle\rho^{1 / 2}\left|-\frac{1}{2}\left(d^{2} / d x^{2}\right)+v(x)\right| \rho^{1 / 2}\right\rangle$ ).

In Fig. 1(e), we demonstrate the effect of increasing the grain size, with $l=1$ and $a=0.6$. When $g=0.25$, the plot is not greatly different to that when $g=0.001$. When $g=1$, and the size of the grain is comparable to the bondlength and atomic size, we observe some critical behavior in $\mu_{\text {grain }}^{n}$; instead of three distinct regions, there are only really two, corresponding to the two atoms. When $g=50$, and the size of the grain is much larger than the system, we lose all information about the atomic and bonding regions; instead, the molecule as a whole, appears as a region of constant local chemical potential. These results show clearly that choosing the grain size changes the resolution at which one views the molecule; for small grain sizes, one sees detailed features, such as atoms and bonds; with much larger grain sizes, one may see the system described in terms of functional groups and larger subunits.

Such analysis may equally be applied to homonuclear systems; there will be seen two atomic regions, symmetrically displaced around a peaked bond region. As the two identical atoms are drawn apart, the two atomic regions tend towards the same constant potential, separated by a peaked "bonding" region which tends toward a delta function. We expect similar results for real three dimensional atoms. The local and subsystem shape chemical potential gives an intuitive picture of the nature of atoms in molecules.

\section{B. Two electron atoms}

We now consider a model in three dimensions, an atom with two noninteracting electrons moving in a central field $v(\mathbf{r})=-Z / r$. Each electron occupies a hydrogenic type orbital $\psi_{n l m}(\mathbf{r})=R_{n l}(\mathbf{r}) Y_{l m}(\theta, \phi)$. We work only with configurations of $s$ type spherical orbitals, ${ }^{16}$ where $l, m=0$ and

$$
\begin{aligned}
& R_{n 0}(r)=C_{n 0}\left(\frac{2 Z}{n}\right) e^{-Z r / n} L_{n}^{1}\left(\frac{2 Z r}{n}\right), \\
& C_{n 0}=-\left[\frac{(n-1) !}{2 n(n !)}\left(\frac{2 Z}{n}\right)^{3}\right]^{1 / 2}, \\
& Y_{00}=\frac{1}{2}\left(\frac{1}{\pi}\right)^{1 / 2} .
\end{aligned}
$$

$L_{n}^{1}$ are associated Laguerre polynomials. For a given $n_{1} s, n_{2} s$ configuration, the density is given by

$$
\rho(\mathbf{r})=\psi_{n_{1} 00}^{2}(\mathbf{r})+\psi_{n_{2} 00}^{2}(\mathbf{r})
$$

This system forms a prototypical model of shell structure, since for $n_{1} \neq n_{2}$, the radial density exhibits oscillations. We hope to pick these up in the shape chemical potential.

The energy, is given by the three dimensional generalization of Eq. (27),

$$
E[\rho, v]=-\frac{1}{2} \int \rho(\mathbf{r})^{1 / 2} \nabla^{2} \rho(\mathbf{r})^{1 / 2} d \mathbf{r}+\int \rho(\mathbf{r}) v(\mathbf{r}) d \mathbf{r} .
$$

We take the subsystem to be a spherical shell grain, of thickness $r=m \rightarrow m+g$. The occupation number of the grain 

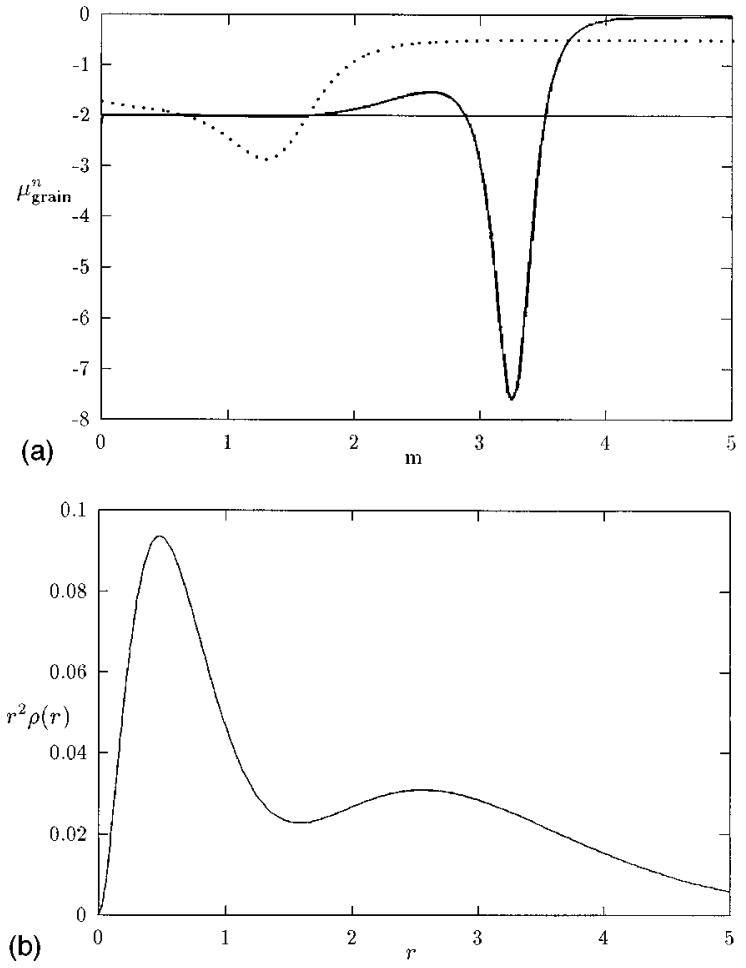

FIG. 2. (a) Two electron atom; plot of $\mu_{\text {grain }}^{n}$, grain size $g=0.001$, for various configurations (thin line, $1 s 1 s$; dotted line, $1 s 2 s$; thick line, $1 s 7 s$ ). See Sec. IV B. (b) Two electron atom; radial density $r^{2} \rho(r)$ in the $1 s 2 s$ atom. Sec. Sec. IV B.

$n(m)$, and the chemical potential $\mu_{\text {grain }}^{n}(m)$, are given by the three dimensional generalizations of Eqs. (29) and (31),

$$
\begin{aligned}
n(m)=\int_{m}^{m+g} 4 \pi r^{2} \rho(r) d r & \\
\mu_{\text {grain }}^{n}(m)= & \frac{1}{n(m)} \int_{m}^{m+g}-\frac{1}{2} \rho^{1 / 2}(\mathbf{r}) \nabla^{2} \rho^{1 / 2}(\mathbf{r}) \\
& +\rho(\mathbf{r}) v(\mathbf{r}) d \mathbf{r} .
\end{aligned}
$$

As in the diatomic, we begin our investigation with a small grain size, $g=0.001$, and $Z=2$. The corresponding $\mu_{\text {grain }}^{n}$ for atoms with configurations $1 s 1 s, 1 s 2 s$, and $1 s 7 s$ is plotted in Fig. 2(a). As expected, the ground state $1 s 1 s$ configuration of the atom, has a constant $\mu_{\text {grain }}^{n}$. When an electron is excited to a higher orbital, however $\mu_{\text {grain }}^{n}$ separates clearly into two shells of different, roughly constant, chemical potential, separated by a sharp barrier in the intershell region. As the second electron is removed to infinity (as in the $1 s 7 s$ atom), $\mu_{\text {grain }}^{n}$ is increasingly constant in the two shells. The barrier between the two approaches a delta function, as the second electron is removed to infinity, reflecting the increasingly rapid change in the derivative of the density between the two regions.

We have, therefore, a well defined partitioning of electrons in space, with one electron in the first shell space, and the second with the other. A check of this proposal is to integrate the density up to the middle of the separating barrier; in the $1 s 7 s$ case, the middle of the barrier is roughly at 3.25 , and the inner region contains almost exactly 1 electron.
The two regions, and the intershell barrier in $\mu_{\text {grain }}^{n}$ agree well with the corresponding regions of the radial density $4 \pi r^{2} \rho(r)$, plotted in Fig. 2(b).

Increasing the grain size has the expected effect. We lose the detail of the intershell regions, and as the grain size increases, $\mu_{\text {grain }}^{n}(m)$ is increasingly flat, losing the distinguishing features of the two shells.

\section{Multielectron atoms}

In the model excited two electron atoms, shell structure can be identified as regions of different constant shape chemical potential. Can this be extended to more complex systems? We attempt to answer that question here. Consider a simple, but essentially correct, representation of the beryllium atom density, in terms of two piecewise exponential shells

$$
\rho(\mathbf{r})= \begin{cases}A_{1} e^{-2 \lambda_{1} r} & 0 \leqslant r \leqslant R \\ A_{2} e^{-2 \lambda_{2} r} & R \leqslant r \leqslant \infty\end{cases}
$$

where the density is continuous, but the derivatives are not. From Wang and Parr, ${ }^{17}$ who used this model in relation to Thomas-Fermi theory, a good representation is given by $A_{1}=354, R=1.096, \lambda_{1}=3.38, \lambda_{2}=0.59$.

For systems with more than two electrons, there exists no satisfactory energy functional of $\rho(\mathbf{r})$ (and its derivatives) alone. ${ }^{1}$ The results in this study will be approximate, and depend strongly on the choice of functional. Nonetheless, we hope to gain some useful insights. The most difficult part of $E[\rho, v]$ is the kinetic energy, for which we use three approximations, giving

$$
\begin{aligned}
& E_{T F}[\rho, v]=C_{F} \int \rho^{5 / 3}(\mathbf{r}) d \mathbf{r}+\int \rho(\mathbf{r}) v(\mathbf{r}) d \mathbf{r}, \\
& E_{v W}[\rho, v]=-\frac{1}{2} \int \rho^{1 / 2}(\mathbf{r}) \nabla^{2} \rho^{1 / 2}(\mathbf{r}) d \mathbf{r}+\int \rho(\mathbf{r}) v(\mathbf{r}) d \mathbf{r}, \\
& E_{T F(1 / 9) v W}[\rho, v]=C_{F} \int \rho^{5 / 3}(\mathbf{r}) d \mathbf{r}-\frac{1}{18} \int \rho^{1 / 2}(\mathbf{r}) \\
& \times \nabla^{2} \rho^{1 / 2}(\mathbf{r}) d \mathbf{r}+\int \rho(\mathbf{r}) v(\mathbf{r}) d \mathbf{r},
\end{aligned}
$$

where $v(r)=-4 / r$ and $C_{F}=\frac{3}{10}\left(3 \pi^{2}\right)^{2 / 3}$. The first, corresponds to the traditional Thomas-Fermi energy functional. ${ }^{18}$ The second is the noninteracting two-electron functional (36), using the von Weizsäcker kinetic energy, ${ }^{19}$ and the third is the well-known Thomas-Fermi-von Weizsäcker model. ${ }^{19}$ In our calculations, we neglect the discontinuity contribution (arising from the discontinuous derivative of the density between shells) to the von Weizsäcker term, as it is small in practice. ${ }^{17}$

The expressions for $n(m)$ and $\mu_{\text {grain }}^{n}$ are given in the previous section by Eqs. (37) and (38). We evaluate the approximate local chemical potential (corresponding to a grain size of 0.001), and plot the results for the three models in Fig. 3. The plots vary significantly with the choice of functional, proof of their very approximate nature. However, two regions of different behavior, clearly corresponding to the 


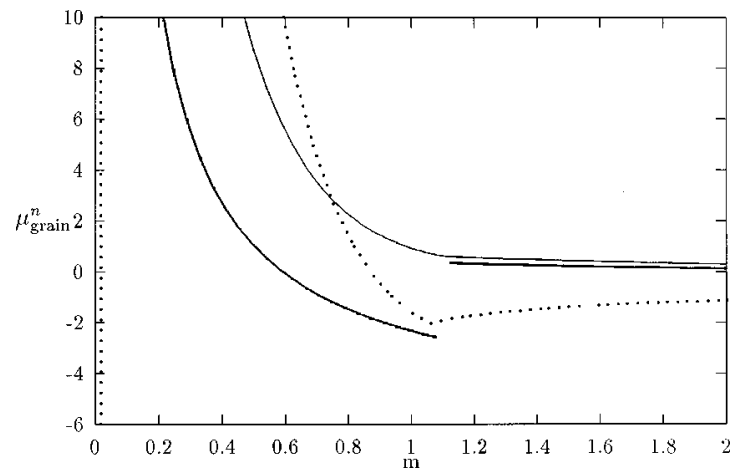

FIG. 3. Be atom $\mu_{\text {grain }}^{n}$, grain size $g=0.001$, using the TF (thin line), vW (thick line), and $\mathrm{TF}_{\overline{9}} \mathrm{vW}$ (dotted line) functionals. See Sec. IV C.

two shells, can be seen, and thus the answer to the question at the start of this section, is in the affirmative. The functionals with the von Weizsäcker contribution give $\mu_{\text {grain }}^{n}$ that is discontinuous, as the derivative of the density changes discontinuously across the shells.

Unlike the two electron atoms, the two shells are not regions of even roughly constant chemical potential. This feature may be due to the approximate nature of the functionals, and must be further investigated.

\section{Comments and some implications for Thomas-Fermi theory}

The above models may be criticized for not evaluating the local chemical potential, at the ground state density corresponding to the energy functional used. For example, in the model two electron diatomic in Sec. IV B, because of the noninteracting nature, the ground-state would correspond to both electrons collapsing onto the more "electronegative", atom. Moreover, the energy functionals used are continuously differentiable, and so regions of different local chemical potential do not exist at the corresponding ground-state densities!

Such comments are strong, but do not invalidate our arguments. The local and subsystem shape chemical potentials are certainly defined at densities other than the groundstate density. We suppose further, that if excited state densities which incorporate important chemical features, reflect themselves in a certain behavior of the local chemical potential, we may expect that ground-state densities which incorporate those same features, have correspondingly similar behavior in the local chemical potentials. To see that this is reasonable, recall the case of a real heteronuclear molecule dissociating to widely separated neutral atoms $\mathrm{X}$ and $\mathrm{Y}$ (the ground state at large bond length). These atoms considered as subsystems, certainly have different subsystems shape chemical potentials (isomorphic chemical potentials). Plotting the local chemical potential will then give a three dimensional step function very similar to that in the one dimensional diatomic model.

We believe that the evidence from the models, while not the last word, is very suggestive of reality. It is clearly desirable to pursue definitive calculations of the local chemical potential in real systems, (within some approximation, such as Hartree-Fock theory).

If we believe that the conclusions drawn from the models do a priori extend to real systems, there are important implications. As stated in Sec. III, a necessary condition for the existence of regions of different local chemical potential at the ground-state density (which we associate with important chemical features) is that the energy functional is not continuously differentiable.

We may then state, with some degree of truth, that in density functional theory, the chemistry lies in the integer discontinuities of $E[\rho, v]$. This may not be so surprising, as ultimately the discontinuities are a manifestation of Fermi statistics, ${ }^{20}$ which is also the physical basis behind the Periodic Table.

Thomas-Fermi-type pure-density theories, explicitly neglect the particle-nature of the density in favor of a statistical model. The inability of such models to reproduce simple chemical features such as shell structure, or the dissociation of a molecule into constituent atoms, may be regarded as due to their continuously differentiable nature which allows the density in different regions to smoothly equilibrate. Turning the argument around, the success of the Kohn-Sham scheme in describing chemistry, may be regarded as due to the correct modeling of the discontinuities.

In this light, attempts to improve the Thomas-Fermi energy functional, by including continuously differentiable functionals of $\rho(\mathbf{r})$ and its gradients, cannot improve its general ability to reproduce chemistry in even a qualitative sense. The inability of recent ${ }^{21}$ and past attempts ${ }^{22}$ to reproduce shell structure, by fitting the functional derivatives of parametrised Thomas-Fermi energy functionals, support this conclusion.

\section{CONCLUSIONS}

In this work, we have introduced the shape chemical potential $\mu_{i}^{n}$ for subsystems, which in the limit of point subsystems is a local chemical potential $\mu^{n}(\mathbf{r})$. We elucidate the basic formal properties of $\mu_{i}^{n}$, demonstrating that due to the complex noncontinuously differentiable nature of the exact energy functional $E[\rho, v]$, this quantity is very different to the system chemical potential $\mu_{0}$. The shape chemical potential $\mu_{i}^{n}$, also does not equalize between subsystems, despite Sanderson's principle. Instead, it is a property that characterizes the electron attracting/donating power of any given density fragment, rather than that of the system as a whole.

We propose that the subsystem chemical potential, and the local chemical potential, may be used to describe chemical processes, especially for local changes in molecules, to metastable states. By looking at the chemical potentials of "grains" of different sizes, we may view the molecule at different resolutions, and thereby identify useful subunits. Very suggestive evidence is provided in model systems, that important chemistry, such as the nature of atoms in molecules, and shell structure, is associated with regions of different local (constant) shape chemical potential.

Such regions of different local chemical potential are not recoverable from Thomas-Fermi theories. We suggest that incorporation of the physical derivative discontinuities of the 
energy functional, for example in the Kohn-Sham scheme, is crucial to recovering chemistry.

It is clearly desirable to further develop these ideas. Calculations of the local chemical potential of real systems are needed to support the assertions of this work. The incorporation of the shape chemical potential into phenomenological chemical theories, through perturbative expansions is important. Also, the formal limiting properties of $\mu_{i}^{n}$, for example in limit of large atomic number, or electron number, should be explored. We believe that by studying this quantity, new insights can be gained into the chemical reactivity.

\section{ACKNOWLEDGMENTS}

G. K.-L. Chan acknowledges Professor J-P. Hansen and R. Daniel for interesting discussions.

${ }^{1}$ W. Yang and R. G. Parr, Density Functional Theory of Atoms and Molecules (Oxford University Press, Oxford, 1989).

${ }^{2}$ R. G. Parr, R. A. Donnelly, M. Levy, and W. E. Palke, J. Chem. Phys. 68, 3801 (1978).

${ }^{3}$ M. Berkowitz, S. K. Ghosh, and R. G. Parr, J. Am. Chem. Soc. 107, 6811 (1984).

${ }^{4}$ R. F. Nalewajski, J. Korchowiec, and A. Michalak, in Topics in Current Chemistry 183, edited by R. F. Nalewajski (Springer, Berlin, 1996).

${ }^{5}$ R. A. Donnelly and R. G. Parr, J. Chem. Phys. 69, 4431 (1978).

${ }^{6}$ J. F. Janak, Phys. Rev. B 18, 7165 (1978).

${ }^{7}$ N. D. Mermin, Phys. Rev. 137, A1441 (1965).

${ }^{8}$ J. P. Perdew, R. G. Parr, M. Levy, and J. L. Balduz, Jr., Phys. Rev. Lett. 49, 1691 (1982)

${ }^{9}$ R. T. Sanderson, Science 121, 207 (1955).

${ }^{10} \mathrm{~J}$-P. Hansen (private communication).

${ }^{11}$ G. K.-L. Chan, J. Chem. Phys. (in press).
${ }^{12}$ W. Kohn, Phys. Rev. B 33, 4331 (1986).

${ }^{13}$ In fact, the original paradox referred to the eigenvalues of the HartreeFock equations, rather than the Kohn-Sham equations, although the spirit of the question is the same.

${ }^{14}$ J. P. Perdew, in Density Functional Methods in Physics, edited by R. M. Dreizler and J. de Providencia (Plenum, New York, 1985). In fact with hindsight, the effective potential investigated by Perdew in this model system, is identical to the negative of the local shape chemical potential, to within an arbitrary constant.

${ }^{15}$ The change in the occupation number of the grain implicitly assumes breaking the continuity of the density, thus taking us out of the regime of $N$-representable densities. In practice, however, we can take an additional limiting process, where at the discontinuity of the density, there is a continuous join by a very steep function (such as a Fermi-Dirac-type function, which tends in the limit to a step function), ensuring the shape of the chemical potential of the grain is well-defined.

${ }^{16} \mathrm{R}$. Mcweeny, Methods of Molecular Quantum Mechanics (Academic, London, 1990).

${ }^{17}$ W-P. Wang and R. G. Parr, Phys. Rev. A 16, 891 (1977).

${ }^{18}$ L. H. Thomas, Proc. Cambridge Philos. Soc. 23, 542 (1927); E. Fermi, Z. Phys. 48, 73 (1928).

${ }^{19}$ E. K. U. Gross and R. M. Dreizler, Density Functional Theory (Springer, Berlin, 1990).

${ }^{20}$ R. K. Nesbet, Phys. Rev. A 56, 2665 (1997); J. P. Perdew and M. Levy, Phys. Rev. B 56, 16021 (1997). Although both works stress the importance of Fermi statistics for derivative discontinuities, we are of the opinion of the latter authors (Perdew and Levy), that the exact density functional itself contains "information about,... Fermi statistics," forgoing the need for extraneous constraints, as in Nesbet.

${ }^{21}$ G. K.-L. Chan (unpublished work). If we fit some functional such that at some particular ground-state density, its functional derivative is exact, then such a functional will have that ground-state density (with shell structure) as an extremal density. But this does not mean that its other extremal densities will exhibit shell structure, for the reasons we argue in this work.

${ }^{22}$ L. J. Bartolotti and P. K. Acharya, J. Chem. Phys. 77, 4576 (1982). 